tionsvoraussetzungen, die der Hausarzt für die Abrechnung erfüllen muss. Hier liefert die einschlägige Stellungnahme der Bundesärztekammer (B̈̈K) vom 20. Juni 2003 einen wertvollen Hinweis. Demnach gibt es in der GOÄ neben den gebietsbezogenen Leistungen auch nicht-gebietsbezogene Sonderleistungen. Nach Auffassung der BÄK bilden lediglich die Abschnitte D bis P der GOÄ jeweils typische Kernleistungen der einzelnen Fachgebiete $a b$.

Die Gebietsgrenzen der Weiterbildungsordnung sind dagegen hier nicht maßgeblich. Zwar darf ein Arzt, der eine Gebietsbezeich- nung führt, nach der Berufsordnung nur innerhalb der Grenzen seines Gebiets tätig werden. Eine generelle Begrenzung der Abrechnungsfähigkeit auf Leistungen des eigenen Fachgebiets lässt sich laut BÄK allerdings aus der GOÄ nicht ableiten.

Nach der geltenden Rechtsprechung verstößt dies auch nicht gegen die Berufsordnung, wie etwa das Schleswig-Holsteinische Oberlandesgericht bereits 1998 klarstellte (Az.: $6 \mathrm{U}$ 48/98). Auch das Amtsgericht München urteilte im Jahr 2002, dass etwaige Beschränkungen aus dem Berufsrecht nicht auf das privatrechtliche Verhältnis zwischen Arzt und
Patient übertragen werden können (Az.: 274 C 18623/02). Diese juristische Betrachtung bedeutet unterm Strich, dass auch z. B. die psychiatrische Untersuchungsleistung nach Nr. 801 von Hausärzten erbracht und abgerechnet werden darf.

Darüber hinaus besteht bei allen oben genannten Abrechnungspositionen in der GOÄ noch die Möglichkeit der Modulation über den Steigerungsfaktor. Wenn z. B. zweiOrgansysteme vollständig untersucht wurden, kann mit entsprechender Begründung die Nr. 7 mit einem höheren Multiplikator in Rechnung gestellt werden.

\title{
Chronikerziffern bei neuen Patienten abrechnen
}

_ Die Nrn. 03220 und 03221 EBM sind nur bei Patienten berechnungsfähig, bei denen mindestens eine lang andauernde, lebensverändernde Erkrankung vorliegt und die Notwendigkeit einer kontinuierlichen ärztlichen Behandlung und Betreuung besteht. Für die Einstufung als "kontinuierliche“ Behandlung muss man laut EBM die letzten vier Quartale betrachten. In mindestens dreien davon muss wegen derselben gesicherten chronischen Erkrankung ein Arzt-PatientenKontakt gemäß Punkt 4.3.1 der Allgemeinen Bestimmungen in derselben Praxis stattgefunden haben.

In mindestens zwei Quartalen muss es ein persönlicher Arzt-Patienten-Kontakt gewesen sein - einer kann also mittelbar, etwa am Telefon oder im Gespräch mit Angehörigen, zustande gekommen sein. Ein Quartal ohne jeglichen Arzt-Patienten-Kontakt ist demnach erlaubt. Das laufende Quartal und somit auch der aktuelle Kontakt zählt bei dieser Betrachtung mit.

Die Abrechnung des Zuschlags nach Nr. 03221 setzt dann einen weiteren persönlichen Arzt-Patienten-Kontakt im laufenden Quartal voraus.

\section{MMW-KOMMENTAR}

Eine kontinuierliche ärztliche Behandlung liegt allerdings auch dann vor, wenn ein chronisch kranker Patient den Hausarzt wechselt. Dann entsteht eine Sondersituation. Der Patient muss vom alten Arzt drei Quartale mit Kontakten "mitbringen", die zur Abrechnung der Nr. 03220 berechtigen. Grund: Laut EBM müssen die Kontakte in derselben Praxis stattgefunden haben. In dem Fall kann der neue Arzt die Nr. 03220 sofort

\section{Beispielfall: Ein neuer Patient}

Ein Patient sieht im August 2016 wegen einer chronischen Erkrankung erstmals seinen neuen Hausarzt. Aus seiner ehemaligen Hausarztpraxis kann folgendes dokumentiert werden:

- 4. Quartal 2015: unmittelbarer Arzt-Patienten-Kontakt

- 1. Quartal 2016: unmittelbarer Arzt-Patienten-Kontakt

- 2. Quartal 2016: mittelbarer Arzt-PatientenKontakt

Die Chronikerpauschale nach Nr. 03220 kann mit der Kennzeichnung " $\mathrm{K}$ " sofort abgerechnet werden, da der Patient die Voraussetzungen aus der alten Praxis mitbringt. abrechnen, muss sie aber mit "K" kennzeichnen. Auch in den beiden folgenden Quartalen kann er so verfahren - bis auch in seiner Praxis drei Quartale erreicht sind. So gesehen wird der Ansatz der Nrn. 03220 und 03221 bei jedem neuen Patienten mit einer oder mehreren chronischen Krankheiten möglich, wenn dieser in den letzten drei Quartalen bei seinem alten Hausarzt die Voraussetzungen erfüllt hat. Der neue Hausarzt kann dann nahtlos weitermachen.

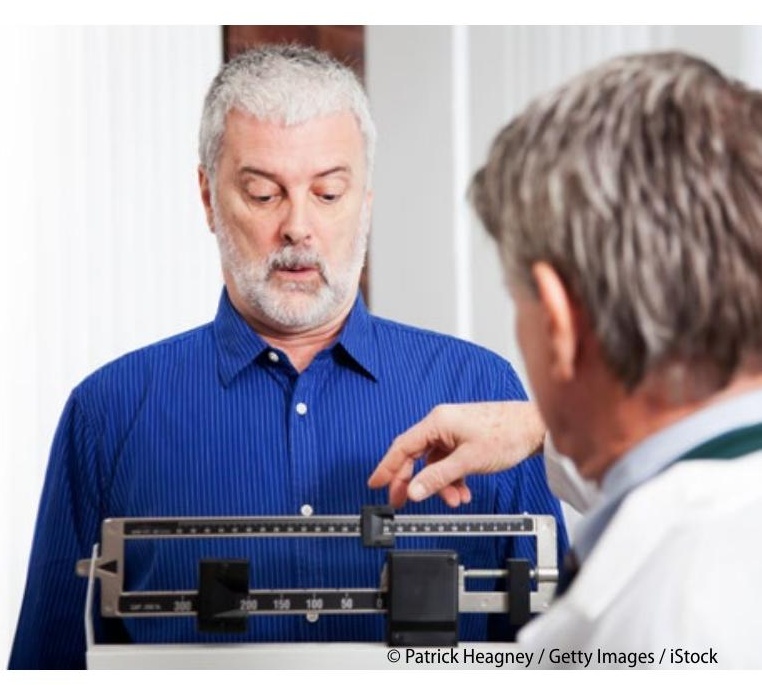

